\title{
Luis Jiménez (dir.), Attention and Implicit Learning
}

"Advances in Consciousness Research » 48, Amsterdam \& Philadelphia, John Benjamins Publishing Company, 2003, 385 p.

\section{Audrey Gerlain}

\section{(2) OpenEdition}

1 Journals

Édition électronique

URL : http://journals.openedition.org/alter/1788

DOI : $10.4000 /$ alter. 1788

ISSN : 2558-7927

Éditeur :

Association ALTER, Archives Husserl (CNRS-UMR 8547)

Édition imprimée

Date de publication : 1 octobre 2010

Pagination : 347-354

ISBN : 2-9522374-6-8

ISSN : $1249-8947$

Référence électronique

Audrey Gerlain, « Luis Jiménez (dir.), Attention and Implicit Learning », Alter [En ligne], 18| 2010, mis en ligne le 01 juin 2020, consulté le 24 septembre 2020. URL : http://journals.openedition.org/alter/1788 ; DOI : https://doi.org/10.4000/alter.1788 


\title{
LUIS JIMÉNEZ (DIR.), ATTENTION AND IMPLICIT LEARNING, «ADVANCES IN CONSCIOUSNESS RESEARCH » 48, AMSTERDAM \& PHILADELPHIA, JOHN BENJAMINS PUBLISHING COMPANY, 2003, 385 P.
}

\author{
Audrey Gerlain
}

"Everyone knows what attention is», déclarait William James dans ses Principles of Psychology ${ }^{1}$. De même, on pourrait dire que chacun sait ce qu'est l'implicite, ce qu'est « apprendre ». Les choses se compliquent lorsqu'il s'agit d'étudier le lien entre "attention » et «implicit learning ». À première vue, définir l'implicit learning comme un processus relativement indépendant de la conscience et de l'attention, éluderait la question sur un tel lien ; or, toute la problématique se centre précisément sur la relativité de cette indépendance. $\mathrm{D}^{\prime}$ autant que la notion jamesienne d'attention est celle d'une attention dérivée, voire d'une distraction, qui rend moins explicites les moments où nous croyons être attentifs. Et la littérature scientifique ne tarit pas à ce sujet, tant sur le phénomène de l'attention, que sur l'idée d'un implicit learning.

C'est ce dont témoigne cet ouvrage dirigé par Luis Jiménez, en articulant la discussion sur l'attention et l'implicit learning autour d'une triple approche qui structure l'ouvrage en trois temps: la discussion cognitive, le débat neuroscientifique y compris computationnaliste, et la mise en perspective phénoménologique (principalement la référence à la phénoménologie de l'attention de William James) qui se déploie à travers d'autres thématiques connexes (la perception, l'orientation, la mémoire). De prime abord, le débat cognitif, qui couvre une soixantaine de pages, paraît moins essentiel que la discussion neuroscientifique, qui occupe la moitié de l'ouvrage, ou que la discussion finale, d'une centaine de pages, sur la relation entre attention et implicit learning. Pourtant, cet encadrement du débat neuroscientifique rend

1. Citation mise en exergue par Tony Lambert dans son article "Visual orienting, learning and conscious awareness" in Luis Jiménez (éd.), Attention and implicit learning, p.253. 


\section{L'attention}

compte du croisement possible entre ces diverses approches, et surtout, de la fertilité de ce croisement pour comprendre ce qu'est l'attention, pour comprendre une phénoménologie de l'attention en dialogue permanent avec les avancées scientifiques.

Les deux premiers articles "Attention and awareness in "implicit" sequence learning » de David R. Shanks, et " Intention, attention, and consciousness in probabilistic sequence learning » de Luis Jiménez, mettent en place les thématiques majeures de l'ouvrage. En premier lieu, il s'agit de discuter la question de l'indépendance de l'implicit learning quant à la conscience et l'attention, véritable fil conducteur problématique de l'ouvrage. Ces études cognitives concernent principalement la question de l'attention dans l'apprentissage d'une seule tâche ou d'une double tâche effectuée, question remobilisée dans les articles suivants. La thèse de D. Shanks est dite sceptique dans la mesure où elle nuance l'idée selon laquelle l'implicit learning ne demande ni aucune ressource attentionnelle, ni aucune conscience, même en son stade minimal d'awareness. L'article de Jiménez a un double intérêt : il propose de démêler différents sens de l'implicit learning, l'un cognitif, les autres non, et établit une troisième voie avec la notion d'attention sélective comme pré-condition à l'implicit learning. Ainsi, dans cet ouvrage, la question des relations réciproques entre implicit learning et attention, est portée par une hypothèse forte, dérivée de la notion jamesienne de l'attention dérivée :

L'attention sélective module ce qui peut être appris implicitement, et l'implicit learning met en forme la façon dont l'attention se déploie ${ }^{2}$.

Ainsi, le débat cognitif ne se distingue pas des deux autres parties de l'ouvrage, mais pose les premiers jalons pour une discussion multidisciplinaire sur des questions récurrentes autour de l'implicit learning et l'attention. En effet, le fil conducteur de l'ouvrage que nous avons identifié, se déploie autour de problématiques connexes telles que la définition de l'implicit learning comme conscience non intentionnelle, sa portée écologique (au sens que lui donne Bateson), ou encore le problème de la reconnaissance d'une qualité implicite de la chose apprise par le sujet. Le débat neuroscientifique et computationnaliste éprouve les hypothèses formulées à ce sujet, par des simulations, des modélisations, des analyses empiriques (à partir de données issues de l'imagerie cérébrale par exemple).

Dans un premier temps, on interroge la structure neuronale de l'implicit learning, et sa possible acception comme catégorie en neurosciences, à travers les articles d'Eliot Hazeltine et Richard B. Ivry « Neural structures that support implicit sequence learning », et de F. Gregory Ashby et Michael B. Casale «The cognitive neuroscience of implicit category learning ». Le premier met en lumière la controverse sur la possibilité ou non de définir une structure

2. Luis Jimenez (éd.), Attention and implicit learning, p. 5. Nous traduisons le passage original: "[...] selective attention does modulate what can be learned implicitly, and implicit learning shapes the deployment of attention". 
neuronale de l'implicit learning. Car les régions cérébrales ne sont pas les mêmes selon le type de tâche apprise, par exemple entre une tâche unique ou double (problème débattu précédemment au niveau cognitif, et formulé cette fois-ci à partir d'études neuroscientifiques). Selon les données fournies par l'imagerie cérébrale, l'activation de certaines régions peut signifier par ailleurs d'autres processus internes, qu'ils soient neurologique, perceptif, moteur, d'encodage, ou de représentation.

Ainsi l'article suivant, d'Ashby et Casale, permet de faire un état des lieux de la "catégorie » de l'implicit learning en neurosciences, tout comme l'article de Jiménez fixait cing acceptions possibles de l'implicit learning. Les auteurs se centrent plus spécifiquement sur la littérature de "catégorisation », i.e. la littérature scientifique qui étudie la façon dont les organismes, même les plus rudimentaires, organisent leurs catégories suivant leur évolution dans un environnement donné, d'où une continuité avec Jiménez, à propos d'une acception « écologiquement valide» de l'implicit learning. La question est de savoir si la catégorie humaine du learning est médiée par un ou plusieurs systèmes de catégorisation du learning. Sans issue définitivement établie, les auteurs choisissent la thématique de la mémoire pour tenter une hypothèse dite "faible » : plusieurs systèmes de mémoire entrent en jeu lors des différents types de tâches qui mobilisent la catégorie du learning. Deux types particuliers de mémoire sont privilégiés, à savoir la mémoire procédurale (dite généralement "mémoire implicite », étudiée par la suite dans l'article 10 de Mulligan et Brown), et les mémoires de la représentation et de la perception. L'idée générale est celle d'un implicit learning indissociable d'une vision du cerveau en réseau d'aires cérébrales en interaction, en réseau de structures neuronales en prise sur l'écologie de l'esprit.

L'article de Peter F. Dominey, "Structure and function in sequence learning: Evidence from experimental, neuropsychological and simulation studies ", ouvre la discussion neuroscientifique et computationnaliste, à la croisée du béhaviourisme et de la neuropsychologie (en étudiant les schizophrènes, ou des malades atteints de Parkinson, ou encore des aphasiques agrammatiques). Le modèle computationnaliste input/output, étayé par des simulations, reprend à nouveaux frais la discussion initiale des premiers chapitres sur le degré d'attention requis dans la tâche unique ou double, tout en prolongeant la question des aires cérébrales activées lors de l'implicit learning. L'article suivant «Temporal effects in sequence learning » d'Arnaud Destrebecqz et Axel Cleeremans, poursuit cette discussion en s'intéressant aux effets temporels de l'implicit learning, notamment les sauts dans la transmission d'informations visibles entre l'explicit learning et l'implicit learning. Ce qui est en jeu, c'est la thématique de la prédiction des prochaines informations à saisir dans l'implicit learning, thématique déjà présente dans l'article de Jiménez et de Dominey. Le dernier article de cette section consacrée à la discussion neuroscientifique et computationnaliste, ancre le problème de l'implicit et de l'explicit learning, au sein d'une vision architecturale 
de la cognition. Dans cette étude de Dieter Wallach et Christian Lebiere, intitulée "Implicit and explicit learning in a unified architecture of cognition ", le propos vise à défendre l'idée d'explicit learning lorsque "déclaration " il y a (ce qui rejoint le propos du dernier article de l'ouvrage sur la verbalisation $\mathrm{du}$ learning), tandis que l'implicit learning constitue un processus, subsymbolique, de calcul des activités et des perceptions du sujet.

La dernière partie de l'ouvrage concerne la discussion sur les relations réciproques entre attention et implicit learning. Après l'aspect temporel, les aspects spatial et contextuel sont étudiés, notamment dans l'article de Tony Lambert "Visual orienting, learning and conscious awareness », et de Yuhong Jiang et Marvin M. Chun «Contextual cueing: Reciprocal influences between attention and implicit learning ". C'est la partie où la notion jamesienne d'attention est mobilisée à plusieurs reprises, i.e. la partie où la discussion est centrée sur la façon dont l'implicit learning trame ou non l'attention. L'article de Tony Lambert est, en ce sens, une excellente mise au point de la conception de l'attention comme attention dérivée de William James. Le paradigme de la vision est également très présent, afin de discuter l'idée d'orientation visuelle qui s'appuie sur une conscience non focale mais périphérique. La notion de cueing vise ainsi à mettre en lumière les indices subtils qui opèrent tels des signaux pour l'orientation de la perception. L'implicit learning peut même conduire à des stratégies attentionnelles, de renforcement de l'attention, ou encore d'une meilleure prise en compte d'événements imprévus. L'implicit learning a des effets sur les représentations, les perceptions, bref le champ expérientiel du sujet, jusque dans l'expérience qu'il a d'être conscient de quelque chose.

De même, la notion de relevance modifie le débat. La question n'est plus de savoir s'il existe un learning plus ou moins explicite, ou absolument implicite ; mais, il s'agit maintenant de se doter d'outils pour accéder à cet implicit learning. Autant les données dans l'imagerie cérébrale, dans les tests de simulation, dans les études cognitives, doivent être "pertinentes » pour la mise au jour d'une structure implicite de la conscience et de l'attention, autant le sujet doit être capable également de « relever » le fonctionnement implicite de sa cognition. Dans les deux derniers chapitres, s'ouvrent alors deux autres thématiques : d'une part, la thématique de la mémoire implicite, avec l'article de Neil W. Mulligan et Alan S. Brown, « Attention and implicit memory", à savoir une mémoire procédurale qui sous-tend chaque processus menant à la tâche apprise; d'autre part, la question de la verbalisation du learning, en particulier lorsque l'implicit learning fait face à une écologie accidentelle, comme le montre l'article de Peter A. Frensch, Hilde Haider, Dennis Rünger, Uwe Neugebauer, Sabine Voigt et Jana Werg, "The route from implicit learning to verbal expression of what has been learned : Verbal report of incidentally experienced environmental regularity ».

En somme, l'implicit learning oscille entre deux pôles, expérientiel et langagier : tantôt expérientiel en tant que processus cognitif neurologique, en prise sur des données écologiques, perceptives, représentationnelles du 
sujet; tantôt langagier en tant que processus de verbalisation et d'expression de la qualité implicite d'un learning au sein d'un explicit learning. L'implicite semble être du côté de l'expérientiel, même si on peut soutenir, à la suite des articles de Wallach et Lebiere, et de Jiménez, que l'implicit learning est en amont de tout explicit learning en ce qu'il est subsymbolique. La discussion à la fois cognitive, neuroscientifique, computationnaliste, et phénoménologique, fournit alors un cadre conceptuel sur l'implicit learning, éprouvé par des modèles, des simulations, des études de cas précises, qui affinent les hypothèses sur sa relation avec l'attention.

Cependant, bien que, dès l'incipit, Luis Jiménez indique la méthode triangulaire «neurosciences, phénoménologie, cognition » (Flanagan), on peut regretter le manque de ressources explicitement phénoménologiques, mis à part William James. Pourtant, le phénoménologue averti reconnaîtra, lors de sa lecture, certains motifs phénoménologiques - la notion de shape qu'on peut traduire maladroitement par celle de "forme » dans la Gestaltheorie, son importance dans la perception, thème phénoménologique par excellence, ou encore la structure husserlienne de la rétention-protention dans le flux temporel de l'attention, même en prise sur un implicit learning. De même, le philosophe trouvera une certaine satisfaction à confronter ces études scientifiques à quelques développements philosophiques sur l'attention, la conscience, l'intention. Car, le lecteur ne doit pas voir dans ce croisement de la phénoménologie avec les neurosciences et la cognition, un effacement de la discussion phénoménologique, ou plus largement philosophique, au profit des neurosciences et de la cognition ${ }^{3}$. Tout au contraire, il s'agit de mettre en place un forum de discussion multidisciplinaire sur l'attention et l'implicit learning, espace de discussion qui occupe la fin de la plupart des articles du volume, et dont on peut reconstituer certaines questions centrales et récurrentes.

Tout d'abord, se pose le problème de l'intention, inauguré par l'article de Jiménez, dans la mesure où l'implicit learning peut être défini comme un processus non intentionnel du learning. Par exemple, la conscience apprend à effectuer une tâche. Si l'on reprend les termes husserliens, la conscience dans l'implicit learning est conscience d'une chose apprise, sans être conscience du processus qui mène à ce résultat. Bien plus, la conscience n'est pas capable d'attribuer cette qualité d'implicite au processus qui sous-tend ce learning, si bien que le sujet a des difficultés à exprimer la chose apprise implicitement. À travers cette question, formulée notamment dans les articles de Jiménez, et de Frensch et al., le phénoménologue retrouvera le débat entre une intentionnalité constituante du premier Husserl, et une intentionnalité latente du dernier Husserl et de sa postérité, particulièrement

3. D'autant qu'on peut rappeler le projet de neurophénoménologie initié par Francisco Varela dans les années 1990. À ce sujet, voir l'article de Claire Petitmengin qui retrace les étapes historiques et épistémologiques de ce projet dont elle fournit un exemple d'application avec son étude des crises épileptiques: «Un exemple de recherche neuro-phénoménologique: l'anticipation des crises d'épilepsie », Intellectica, 2005/1, 40, p. 63-89. 


\section{L'attention}

Merleau-Ponty. Cette conception de la conscience résulte d'une plus grande prise en compte de la relation entre la conscience et le monde, radicalisée dans l'implicit learning et l'attention, dès lors qu'on adopte le paradigme écologique, comme le fait Jiménez dans son article sur l'intention, l'awareness et l'implicit learning.

Nous avons également amorcé à plusieurs reprises le problème du langagier face à l'expérientiel, d'autant que la thèse de l'ouvrage est de montrer en quoi l'implicit learning est un processus en prise sur les représentations, les perceptions et le comportement de l'individu. Or, toute la question est, premièrement, celle de savoir comment le sujet peut avoir accès à cette dimension tacite de l'expérience, comment il peut en rendre compte verbalement, puis celle, plus épistémologique et heuristique, de savoir quels outils sont disponibles pour étudier la structure neuronale, le fonctionnement béhavioriste et le processus cognitif, à l'œuvre dans l'implicit learning. Luis Jiménez opte pour une distinction entre l'acquisition de l'implicit learning, et l'expression par le sujet qu'une chose a été apprise implicitement. Le débat autour de l'implicit learning, et ses pôles expérientiel ou langagier, rend compte de toute la distinction philosophique entre le fait et la procédure, le quoi et le comment : à savoir, le quid et le quo modo des Latins, le know what et le know how des Anglais, ou le wissen et können des Allemands.

On peut mettre également en perspective l'implicit learning quant à la notion de tacit knowing forgée par Michael Polanyi dans The Tacit Dimension ${ }^{4}$. En effet, ce scientifique américain devenu par la suite épistémologue, est une référence incontournable dès lors qu'on tente de reconstituer un des jalons historiques de la notion d'implicite dans la littérature scientifique et philosophique. Le tacit knowing signifie ce processus d'émergence du sens et de découverte, en particulier pour un chercheur, à partir d'une dimension tacite de la perception des choses et de la manière de conduire sa recherche. Polanyi définit le tacit knowing selon l'idée du «we know more than we can $t_{e l l}{ }^{5}$ : autrement dit nous en savons plus que nous ne pouvons en dire, de la même manière que notre corps en fait plus que ce que nous pourrions en faire consciemment. Notre connaissance ne s'explique pas par l'attention explicite que nous portons sur un objet présent, mais bien plus par sa manifestation en tant qu'objet implicite de découverte ${ }^{6}$. Il se propose de rendre compte de ce mécanisme du tacit knowing au cœur d'une théorie de la perception pour toucher aux structures même de l'esprit dans l'appréhension de la réalité. Selon celle-ci : 1) le chercheur se concentre sur son sujet de recherche, c'est le terme proximal de sa perception ; 2) dans un même temps toute sa recherche est travaillée par divers éléments aperçus implicitement,

4. Michael Polanyi, The Tacit Dimension, Gloucester (Mass.), Peter Smith, 1983.

5. Ibid., p. 4

6. Ibid., p.11 "We may say, in general, that we are aware of the proximal term of an act of tacit knowing in the appearance of its distal term; we are aware of that from which we are attending to another thing, in the appearance of that thing". 
sans qu'il en soit conscient de manière thétique, et qui, pourtant, orientent sa recherche et ses résultats : c'est le terme distal.

Dans l'ouvrage de Jiménez, nous retrouvons ces intuitions philosophiques de Polanyi, tout en cristallisant le débat autour du lien entre cette dimension implicite de la connaissance et l'attention. Car ce que Polanyi définit en termes de conscience focale et distale, l'ouvrage Attention and Implicit learning le remobilise à travers la notion d'attention dérivée, ou divisée, de James. Toutefois, Polanyi récuse la conception jamesienne de la conscience comme franges. Par ailleurs, les mêmes champs thématiques sont privilégiés, à savoir ceux de la cognition, la perception, de la représentation et de l'écologie. Cependant, du tacit knowing à l'implicit learning, c'est un pas supplémentaire vers la connaissance du know how qui est franchi. Certes, pour Polanyi, le tacit knowing élucide la logique de la découverte en science, celle de l'apprentissage en gnoséologie, i.e. le problème platonicien du Ménon. Mais le knowing semble comparativement plus statique et normatif que le learning. Car, rétrospectivement, l'implicit learning paraît plus ancré dans la dynamique processuelle du know how en ce qu'il implique une dimension de pratique avec les notions de performance, d'entraînement, d'effort répété.

Dans ce parcours tour à tour philosophique, scientifique, phénoménologique, il est intéressant de noter la façon dont la réflexion sur l'attention modifie celle sur la conscience, puis la façon dont l'implicit learning modifie la conception de l'attention et de la conscience. Déjà le champ de l'attention dérivée pour James, sélective pour Jiménez, distale pour Polanyi, modifie la pensée de la conscience en une pluralité de tâches effectuées, de perceptions, de processus internes, qui sous-tendent toujours déjà le flux de l'activité consciente. Mais, qui plus est, la structure implicite implique un processus relativement indépendant à la fois de la conscience et de l'attention. Du fait que l'implicit learning suppose des tâches différentes, des systèmes de mémoire différents, des changements dans l'activation des aires cérébrales, il nuance l'activité de la conscience focale, et renforce la question de l'attention dans son acception comme attention dérivée, sélective. Car, attention et conscience sont souvent vues comme synonymes, ce que rappelle très bien l'article de Tony Lambert. Or, dès lors que l'attention est assumée comme dérivée et sélective, elle étaye l'idée de processus implicites, i.e. distaux, dans le fait d'avoir conscience de quelque chose, de l'apprendre. Mais cela suppose de distinguer l'acquisition d'un implicit learning et la possible expression qu'une chose a été apprise implicitement. Ainsi, « attention » et implicit learning participent de l'avancée des débats sur la conscience.

De plus, penser l'attention en lien avec l'implicit learning suppose de déplacer la problématique de l'attention dérivée vers ce qu'elle implique comme performance de la part du sujet. C'est la qualité d'implicite qui permet de déployer l'attention dans la part d'effort, d'entraînement, de répétition et de performance, que suppose le learning, et ce, en fonction de la 


\section{L'attention}

pluralités de tâches à apprendre, de la capacité à pouvoir verbaliser cet apprentissage implicite. L'étude de l'attention suppose une mise en abyme lorsqu'on l'articule à l'implicit learning: se rendre attentif aux processus non explicites du «se rendre attentif à ». L'attention, la conscience et l'explicite sont des synonymes relatifs, ce que l'implicit learnig ébranle, nuance, ou du moins, invite à discuter: un espace de discussion coordonné par Luis Jimenez, car Attention and implicit learning devient le forum de discussion privilégié pour tout lecteur préoccupé par ces questions. 